\title{
Comparison Between Microscopic and Rapid Test Assay in the Detection of Malaria Parasite Infection in Patient Attending Some Hospitals in Sokoto Nigeria
}

\author{
Garba Ibrahim, ", , Umar Asiya Imam', Ganau Ahmed Mohammed", \\ Raji Mudasiru Iyanda Omowale ${ }^{2}$, Fana Sani Abdullahi ${ }^{1}$, Shinkafi Sa'adatu Aliyu³, \\ Kazeem Ademola ${ }^{1}$ \\ ${ }^{1}$ Department of Medical Microbiology, School of Medical Laboratory Science, Usmanu Danfodiyo University, Sokoto, Nigeria \\ ${ }^{2}$ Department of Pharmaceutics and Pharm, Microbiology, Usmanu Danfodiyo University, Sokoto, Nigeria \\ ${ }^{3}$ Department of Biological Sciences, Federal University, Gusau, Nigeria
}

Email address:

ibrahimzurmi@yahoo.com (G. Ibrahim)

${ }^{*}$ Corresponding author

\section{To cite this article:}

Garba Ibrahim, Umar Asiya Imam, Ganau Ahmed Mohammed, Raji Mudasiru Iyanda Omowale, Fana Sani Abdullahi, Shinkafi Sa'adatu Aliyu, Kazeem Ademola. Comparison Between Microscopic and Rapid Test Assay in the Detection of Malaria Parasite Infection in Patient Attending Some Hospitals in Sokoto Nigeria. American Journal of Biomedical and Life Sciences. Vol. 8, No. 4, 2020, pp. 64-68. doi: 10.11648/j.ajbls.20200804.11

Received: March 18, 2020; Accepted: April 14, 2020; Published: June 9, 2020

\begin{abstract}
Malaria is a serious public health problem, this study was aimed at comparing Microscopy and Rapid Diagnostics test in malaria parasite detection in patients in some hospitals in Sokoto, Nigeria. Blood Samples were collected by venipuncture and dispensed into EDTA bottle. Thick blood films were made by adding few drops of anticoagulated blood on a clean glass slides and emulsifying to coin size using a spreader and allowed to air-dry and stained with $10 \%$ Geimsa stain for 10 minutes, allowed to air-dry and examined using $\times 100$ objective to confirm the presence of malaria parasites. Of the 100 patients screened, $37 \%$ and $24 \%$ were positive for malaria using Carestart and SD-Bioline, while $53 \%$ were malaria positive by microscopy. Prevalence of malaria parasite by sex was $53.6 \%$ and $52.3 \%$ in females and males. Malaria prevalence by age was highest among patients aged 11-20, while the lowest prevalence was observed in patients aged 51-60 years. Based on occupation, the prevalence of malaria was highest among unemployed patients $58.5 \%$, followed by $22.6 \%$ prevalence of malaria among business men and women. No significant difference was observed in malaria infection by sex, age and occupations $\mathrm{P}<0.05$. The sensitivity and specificity of Carestart and SD-Bioline screening kits were 54.7\% and 82.6\%, 34.6 and $87.2 \%$. The predictive positive value of Carestart was $78.4 \%$ while its negative predictive value was $61.3 \%$ in contrast to SD-Bioline with $75 \%$ and $54.7 \%$ as the positive predictive value and negative predictive value. Rapid diagnostic test are suitable alternatives to microscopy particularly in resources limited rural areas.
\end{abstract}

Keywords: Comparison, Microscopic, Rapid Test, Malaria, Sokoto

\section{Introduction}

Malaria remains an important parasitic disease worldwide [1]. The most ubiquitous and deadly species is the Plasmodium falciparum [2]. It is prevalent in tropical regions because the significant amount of rainfall, consistently high temperatures and high humidity, along with stagnant waters in which mosquito larvae readily mature, provide them with the environment they need for continuous breeding [3]. Management of malaria requires prompt diagnosis of malaria by microscopy, immunochromatographic rapid assay or other available tools [4]. Rapid diagnostic test is a method that detects malaria antigen in a small amount of blood, usually 5$15 \mu \mathrm{L}$, by immunochromatographic assay with monoclonal antibodies directed against the target parasite antigen and impregnated on a test strip [4]. The diagnosis of malaria in 
clinical laboratories mainly depends on blood smear microscopy and this technique remains the most widely used [5]. Microscopic examination of blood is the most affordable, accessible, widely used and reliable technique for diagnosis of malaria infection [5]. Giemsa microscopy is regarded as the most suitable diagnostic technique for malaria control because it is inexpensive to perform, able to differentiate malaria species and quantify parasites [6]. Examination using Giemsa-stained thick blood films remains the reference standard for detection of malaria parasites [7] and cause significant reduction in referrals and in patient's length of hospital stay [7]. Preferred targeted antigens for RDTs are those which are abundant in all asexual and sexual stages of the parasite. Currently the focus of RDT is on the detection of Histidine-Rich Protein2 (HRP-2) from Plasmodium falciparum and Parasite-Specific Lactate Dehydrogenase (pLDH) or Plasmodium aldolase from the parasite glycolytic pathway found in all species. However, several factors in the manufacturing process as well as environmental conditions may affect RDT performance, and these include suboptimal sensitivity at low parasite densities, inability to accurately identify parasites to the species level or quantify infection density, and a higher unit cost relative to microscopy [8]. RDTs are antigen capture tests that have been shown to be capable of detecting $>100$ parasites $/ \mu \mathrm{L}(0.002 \%$ parasitemia $)$ [8]. The tests that utilize PfHRP2 have been found to be more sensitive than pLDH based ones, especially at low parasite densities, with certain exceptions. Both PfHRP2 and pLDH RDTs have been found to be more sensitive than aldolasebased tests. The PfHRP2 based RDTs can detect antigen when $P$. falciparum parasites are sequestered either in placental tissues or elsewhere, which makes them not to be present in peripheral blood for detection by microscopy [9].

The use of rapid diagnostic tests is suggested to have largest impact. Its implementation has been reported to be cost effective [10]. It is an immune-chromatographic capture procedure which targets antigens abundant in all asexual and sexual stages of the parasite.

\section{Materials and Methods}

\subsection{Study Area}

Sokoto is a city located in the extreme northwest of Nigeria, near the confluence of the Sokoto River and the Rima River. As of 2006 it has a population of 427,760. Sokoto is the modern-day capital of Sokoto State. It lies between longitudes $0.5^{\circ} .11^{\prime}$ to $13^{\circ} .03^{\prime}$ east and latitude $13^{\circ} .00$ to $13^{\circ} .06$ North GPS coordinates for Sokoto www.google.com

Specialist Hospital Sokoto and Maryam Abacha women and children Hospital are government owned Hospital located in Sokoto south Local Government Area of Sokoto state, Nigeria.

\subsection{Study Population}

The study was hospital based and involved hundred (100) blood samples collected from patients aged 0-70 presenting at Specialist Hospital Sokoto (SHS) and Maryam Abacha Women and Children Hospital.

\subsection{Ethical Consideration}

Ethical clearance was obtained from the ethical review committee in Specialist Hospital and Maryam Abacha Women and Children Hospital Sokoto.

\subsection{Inclusion Criteria}

Patients aged 0-70 with symptoms suggestive of malaria presenting to the hospital and referred to the laboratory for malaria parasite examination were used for the study. Verbal informed consent of the subjects enrolled for the study was also sought.

\subsection{Exclusion Criteria}

Those patients on malaria medication and those with other symptoms not suggestive of malaria were excluded from the study.

\subsection{Study Design}

Cross-sectional comparative study: that is aimed at comparing between the efficiency of microscopic and immunochromatographic rapid assay methods in the diagnosis of malaria infection.

\subsection{Microscopic Examination}

Samples for microscopy were collected through venous blood technique and dispensed into EDTA bottle. Thick blood films were made by adding few drops of EDTA anticoagulated blood on a clean grease-free glass slides, then emulsifying to coin size using tip of a spreader to cover about $15 \times 15 \mathrm{~mm}$ and allowed to air-dry. Then stained with $10 \%$ Geimsa stain for 10 minutes and allowed to air-dry and examined by light microscope using oil immersion objective $(\times 100)$ to confirm the presence of malaria parasites.

1. 1-10 parasites per hundred (100) high power fields $(\mathrm{HPF})=(+)$

2. 11-100 parasites per hundred (100) high power fields $(\mathrm{HPF})=(++)$

3. 1-10 parasites per every high powerful field $(\mathrm{HPF})=(+++)$

4. More than 10 parasites per every high power field $(\mathrm{HPF})=(++++)$

5. If no parasite is found after examination of hundred (100) high power fields $(\mathrm{HPF})=$ Negative [11].

Parasitaemia was estimated on all positive slides using the method described above.

\subsection{Malaria RDT}

Five (5) $\mu \mathrm{L}$ of blood was used to screen for malaria using the CareStart and SD-Bioline malaria RDT kit, following the manufacturer's instructions. This RDT is a qualitative immunochromatographic test that detects P. falciparum HRP- 
II and Plasmodium lactate dehydrogenase, which is a glycolytic enzyme common to $P$. falciparum, Plasmodium ovale, Plasmodium vivax and Plasmodium malariae asexualstage parasites. Whole blood $(5 \mu l)$ from the patients was taken using pipette and dispensed into sample region labeled "S". Then $60 \mu 1$ of buffer solution was added into "A" well. The result was read after 20 minutes according to manufacturer's instruction

\subsection{Interpretation of the Test Result}

a) Positive: The presence of two colour bands (one band in the control " $\mathrm{C}$ " region and another band in the test " $\mathrm{T}$ " region indicates a positive result.

b) Negative: The presence of only one band in the control "C" region indicates a negative result.

Invalid: The absence of band in the control " $\mathrm{C}$ " region indicates invalid result.

SPSS version 20 statistical data editor software was used for entering, storage and analyses of data. Chi-square $\left(\chi^{2}\right)$ test was used to find statistical association between the variables.

\section{Results}

Out of 100 stained thick blood film examined, 53 (53\%) were positive for malaria infection using microscopic technique while 37 (37\%) patients were positive for malaria as detected by Carestart RDT kit, in contrast to 24 (24\%) patients that tested positive for malaria parasite infection with SD-Bioline kit as presented in Table 1. Of the One hundred (100) thick stained blood film examined, twenty-three $(43.4 \%)$ male patients tested positive for malaria infection while thirty $(56.6 \%)$ females also tested positive for malaria parasite. Statistical analysis showed no significant association in malaria infection between the gender ( $\mathrm{P}$ Value $>0.005$ ) Table 2 . The prevalence of malaria infection by age is presented in Table 3. The highest prevalence 19 (35.8\%) was observed among patients aged 11-20, whereas $15(28 \%)$ prevalence of malaria infection was observed among 21-30 age group while the least prevalence $1(1.8 \%)$ was observed among age group 51-60, there was statistically significant association between the age and malaria infection among the patients. (p. value $\left.=0.002, \chi^{2}=27.90\right)$. The prevalence of malaria infection on the basis of occupation is shown in Table 4. The highest prevalence of malaria infection $(58.5 \%)$, was observed among unemployed patients while the lowest prevalence of malaria $(5.7 \%)$ was observed among farmers. Patients whose occupation is business and Civil servant had $22.6 \%$ and $13.2 \%$ prevalence of malaria infection respectively. Statistical analysis showed association between malaria infection and occupation. (p. value $=0.021$, $\chi^{2}=12.92$ ).

The result of the evaluation of Rapid Diagnostic Test using microscopy as the gold standard in the diagnosis of malaria parasites. The sensitivity of Carestart kit was $54.6 \%$ while SD-Bioline had a sensitivity of $34.6 \%$. The specificity of SD-Bioline was $87.2 \%$ while the Carestart kit had a specificity of $82.6 \%$. The positive predictive value of
Carestart kit was $78.4 \%$ and a negative predictive of $61.3 \%$ in contrast to the SD-Bioline with a predictive positive value of $75.0 \%$ and a predictive negative value of $54.7 \%$ respectively (Table 5).

Table 1. Malaria Parasites Prevalence by Microscopy and RDT's kits among patients.

\begin{tabular}{lll}
\hline Techniques & Positive samples & Prevalence (\%) \\
\hline Microscopy & 53 & $53 \%$ \\
Carestart & 37 & $37 \%$ \\
SD-Bioline & 24 & $24 \%$ \\
\hline
\end{tabular}

$\mathrm{n}=$ Number of samples examined $(\mathrm{n}=100)$.

Table 2. Prevalence of Malaria Parasites Infection among patients based on Gender.

\begin{tabular}{lllll}
\hline Gender & $\begin{array}{l}\text { Number } \\
\text { examined }\end{array}$ & $\begin{array}{l}\text { Number } \\
\text { positive }\end{array}$ & Prevalence & P-value \\
\hline Male & 44 & 23 & $52.3 \%$ & $0.199^{*}$ \\
Female & 56 & 30 & $53.6 \%$ & \\
Total & 100 & 53 & $53 \%$ & \\
\hline
\end{tabular}

*Values differ significantly $(\mathrm{p}<0.05)$ between male and female $\left(\chi^{2}=0.0166\right)$.

Table 3. Prevalence of Malaria Parasites Infection based on Age group.

\begin{tabular}{lllll}
\hline Age group (Yrs) & $\begin{array}{l}\text { Number } \\
\text { examined }\end{array}$ & $\begin{array}{l}\text { Number } \\
\text { positive }\end{array}$ & Prevalence & P-value \\
\hline $0-10$ & 11 & 10 & $18.9 \%$ & $0,002 *$ \\
$11-20$ & 25 & 19 & $35.8 \%$ & \\
$21-30$ & 31 & 15 & $28.3 \%$ & \\
$31-40$ & 18 & 2 & $3.8 \%$ & \\
$41-50$ & 9 & 4 & $7.5 \%$ & \\
$51-60$ & 4 & 1 & $1.9 \%$ & \\
$61-70$ & 2 & 2 & $3.8 \%$ & \\
Total & 100 & 53 & $100 \%$ & \\
\hline
\end{tabular}

*Values differ significantly $(\mathrm{P}<0.05)$ between age group $\left(\chi^{2}=27.9\right)$.

Table 4. Prevalence of Malaria Parasites Infection based on Occupation.

\begin{tabular}{lllll}
\hline Occupation & $\begin{array}{l}\text { Number } \\
\text { examined }\end{array}$ & $\begin{array}{l}\text { Number } \\
\text { positive }\end{array}$ & \% prevalence & P-value \\
\hline Civil servant & 10 & 7 & $13.2 \%$ & $0.021^{*}$ \\
Business & 34 & 12 & $22.6 \%$ & \\
Farming & 11 & 3 & $5.7 \%$ & \\
Unemployed & 45 & 31 & $58.5 \%$ & \\
Total & 100 & 53 & $100 \%$ & \\
\hline
\end{tabular}

*Value differ significantly $(\mathrm{P}<0.05)$ among occupation $\left(\mathrm{Df}=3, \chi^{2}=12.9\right)$.

Table 5. Evaluation of RDTs Using Microscopy as Gold Standard.

\begin{tabular}{llllll}
\hline RDTs & Sn (\%) & Sp (\%) & PPV (\%) & NPV (\%) & P-value \\
\hline Carestart & $54.7 \%$ & $82.6 \%$ & $78.4 \%$ & $61.3 \%$ & 0.000 \\
SD-Bioline & $34.6 \%$ & $87.2 \%$ & $75.0 \%$ & $54.7 \%$ & 0.000 \\
\hline
\end{tabular}

$\mathrm{Sn}=$ Sensitivity, $\quad \mathrm{Sp}=$ Specificity, $\quad \mathrm{PPV}=$ Positive $\quad$ Predictive Value, $\mathrm{NPV}=$ Negative Predictive Value.

\section{Discussion}

Malaria is one of the most important public health problems in the world [12]. Endemic malaria, population movements, and foreign travel all contribute to the malaria diagnostic problems faced by the laboratory that may not have appropriate microscopy expertise available [13]. In this 
study, the prevalence of malaria parasite by microscopic technique was $53 \%$, while Carestart kit was $37 \%$ and SDBioline $24 \%$. The observed high prevalence of malaria by microscopic method may be attributed to precision of the method compare to RDTs, similarly, RDTs detects parasite antigens which may be error prone probably because of low sensitivity of RDTs at low parasitaemia compared to microscopy. The findings in this study is in agreement with $52.9 \%$ malaria detection by microscopy and $42.6 \%$ using the Carestart kit as reported by Sheyin [14].

The observed high prevalence of malaria parasite infection in female in this study is probably because of lack of preventive measures by the patients screened as most of them were from low income settings and living in area of malaria endemic area are prone to infection.

High prevalence of malaria also observed in this study among the patients aged 11-20 (35.8\%) followed by aged 21 $30(28.3 \%)$ then aged $0-10$ has $18.9 \%$ while the least infection rate was observed among aged 51-60 (1.9\%). The high prevalence of malaria among aged 11-20 may probably due to their geographical area which is endemic and too much exposure to malaria vector (female anopheles' mosquito) as a result of improper drainage systems. Out of the 11 patients, 10 tested positive for malaria parasite infection, this is attributed to the fact that children born to immune mothers are protected against malaria disease during their half years of life by maternal antibodies, this passive immunity is followed by 1 or 2 years of increased susceptibility before acquisition of active immunity which occurred after repeated exposure to malaria infection [15].

The observed high prevalence of malaria parasite infection among unemployed patients in this study (58.5\%) is probably because of poverty, they couldn't afford to purchase materials for preventive measure like: mosquito net, mosquito repellant and insecticide.

In this study, CareStart kit had sensitivity of $54.7 \%$, specificity of $82.6 \%$, positive predictive value (PPV) of $78.4 \%$, and negative predictive value (NPV) of $61.3 \%$ while SD Bioline kit had sensitivity of $34.6 \%$, specificity of $87.2 \%$, PPV of $75.0 \%$, and NPV of $54.7 \%$. It is expected that any RDT used for malaria diagnosis should have a high sensitivity of $95 \%$ and specificity $97 \%$ [16]; in this study routine microscopic examination of Giemsa-stained blood smears considered as the gold standard for malaria diagnosis had a sensitivity of $77.2 \%$ and was able to detect more parasites than the RDT (sensitivity 62.3\%). Though the specificity of microscopy $(72 \%)$ was not as high as that of RDT (87.4\%); nevertheless, it has high sensitivity, possibility for quantification of parasitemia, and easy handling which is a good advantage. Detection of parasites depends on several factors including the volume of blood screened and the experience of the microscopist, among others. Equally, the information obtained by microscopy is limited when parasite levels are very low or when parasite morphology is altered [17]. The development of rapid diagnostic assays has attempted to address some of these shortcomings of microscopy. RDTs have the potential to improve the accuracy and time needed for malaria diagnosis particularly for laboratories in low or nonendemic countries, where expertise with microscopy may be limited. Major advantages of RDTs include the fact that it can be performed close to home in settings with no sophisticated infrastructure, and they do not require much skill although some level of training is needed in order for RDTs to be used properly. This is in contrast with the RDTs results in this study.

The false negative (FN) of Carestart and SD-Bioline kits in this study were $24(46.2 \%)$ and $34(64.2 \%)$ respectively using microscopy as the gold standard similar to findings by (17) due to lack of sensitivity of RDTs at low parasitaemia compared to microscopy.

The false positive rate in this study for the Carestart and SD-Bioline kits are $8 \%$ and $6 \%$ respectively. This could possibly be explained by the fact that patients who were possibly already on treatment and whose parasitaemia has cleared could still have tested positive by the rapid diagnostic test picking up remnant antigens [18]. It could also occur as a result of the sequestration of parasitized erythrocytes in tissue capillaries and placental malaria in the absence of peripheral blood parasitaemia may have also yielded positive RDT and negative microscopy for malaria parasites $[19,20]$. There is also a reported cross reactivity between RDTs that detect histidine-rich protein 2 and rheumatoid factor, which gives a false positive result for malaria parasites [20].

RDTs can be useful in screening febrile returnees from endemic areas and they are recommended in situations exceeding microscopy capability such as in an outbreak or in occupationally exposed groups [21]. As RDTs improve, including in sensitivity and in ability to measure parasitaemia levels, at least semi-quantitatively, the scope of RDT application will expand. Current RDTs cannot replace microscopy; other factors such as the quality of the products, storage temperature and humidity, and end users' performance can affect the diagnostic accuracy. Although RDTs are used as diagnostic methods, diagnosis by microscopy should never be abandoned because it is the gold standard in endemic areas. In addition, microscopy allows the calculation of parasitic densities and identification of all species and is cheaper than the other methods [22]. Malaria diagnostics should be performed immediately on suspicion of malaria and the gold-standard is microscopy of Giemsastained thick and thin blood films [23]. Accurate diagnosis of malaria is important for effective disease management and control [24].

\section{Conclusion}

Quality rapid diagnostic test methods are accurate and suitable alternatives to microscopy particularly in resources limited settings devoid of electricity and experienced microscopist as it assists in scaling up the coverage of parasite- based diagnosis. 


\section{Conflict of Interest}

The authors declare that they have no competing interests.

\section{Funding Source}

No financial support was given by any individual or organization.

\section{Acknowledgements}

We wish to thank the management of Usmanu Danfodiyo University Sokoto, Specialist Hospital and Maryam Abacha Women and Children Hospital Sokoto, North western Nigeria for their cooperation in this study.

\section{References}

[1] World Health Organization: A global strategy for malaria control Geneva WHO. TDR/WHO-WPRO 2014.

[2] Sani, A. F., Asiya, U. I., Mohammed, D. A. B., Anka, S. A and Shehu, U. N Prevalence and risk factors associated with malaria infection among pregnant women in semi-urban community of north-western Nigeria Infect dis of Pov, 2015; 4: 24.

[3] Collins, W. E., "Plasmodium knowlesi: a malaria parasite of monkeys and humans" Annual Rev of Ent., 2012; 57: 107-121.

[4] Wongsrichanalai, C., Barcus, M. J., Muth, S., Sutamihardja, A and Wernsdorfer, W. H A review of malaria diagnostic tools: Microscopy and rapid diagnostic test (RDT). anthones inhibitors of $\alpha$-glucosidase and glycation from Garcinia nobilis Am J of Trop and Med Hyg., 2007;. 77: 119-127.

[5] Biadglegne, F., Belyhun, Y., Ali, F., Walle, F., Gudeta, N., Kassu, A and Mulu, A Does the practice of blood film microscopy for detection and quantification of malaria parasites in northwest Ethiopia fit the standard? BMC Health Serv Res., 2014; 14: 529.

[6] Barnish, G., Bates, I and Iboro, J Newer drug combinations for malaria Mal J., 2004; 328: 1511-1512.

[7] Boyce, R. M., Muiru, A., Reyes, R., Ntaro, M., Mulogo, E., Matte, $\mathrm{M}$ and Siedner, M. J., Impact of rapid diagnostic tests for the diagnosis and treatment of malaria at a peripheral health facility in Western Uganda: An interrupted time series analysis Mal J., 2015; 14: 317-335.

[8] Ojurongbe, O., Adegbosin, O. O., Taiwo, S. S., Terry Alli, O. A., Olowe, O. A., 1 Taiwo Adetola Ojurongbe, T. A., Bolaji, O. S and Adeyeba, O A Assessment of Clinical Diagnosis, Microscopy, Rapid Diagnostic Tests, and Polymerase Chain Reaction in the Diagnosis of Plasmodium falciparum in Nigeria Mal Res and Treat., 2013; 5 pages.

[9] Garba, B. I., Muhammad, A. S., Musa, A., Edem, B., Yusuf, I., Bello, N. K., Adeniji, A. O and Kolawole, T Diagnosis of malaria: A comparison between microscopy and rapid diagnostic test among under-five children at Gusau, Nigeria Sub-Saharan Afr J Med 2016; 3: 96-101.

[10] Batwala, V., Magnussen, P., Hansen K. S and Nuwaha, F) Cost-effectiveness of malaria microscopy and rapid diagnostic tests versus presumptive diagnosis: Implications for malaria control in Uganda Mal J, 2011; 10 407-419.

[11] Cheesbrough, M District laboratory practice in tropical countries part 1, second edition, published in U.S by Cambridge University press, 2005; pp 229-243.

[12] Khanam, S Prevalence and Epidemiology of Malaria in Nigeria: A Review Int $J$ of Res in Pharm and Biosci., 2017; 4 (8): 10-12.

[13] Moody, A Rapid Diagnostic Tests for Malaria Parasites, Clin Microb Rev 2002; 15 (1): 66-78.

[14] Sheyin, Z and Bigwan, I. E Comparison of CARE START HRP2 rapid malaria test with light microscopy for guiding patient's treatment of fever in Nigerian endemic areas $J$ of Med and Med Sci., 2013; 4 (9): 353-356.

[15] Permal, K. L, Bell, D. R., Jorgensen, P and Christopel, E. M Malaria risk; estimation of the malaria burden Nature, 2005; 437: E3.

[16] WHO Malaria Rapid Diagnosis: Making It Work World Health Organization, Manila 2003.

[17] Jamshaid, I and Nabila, K Comparison of two commercial assays with expert microscopy for confirmation of symptomatically diagnosed malaria $\mathrm{Am} \mathrm{J}$ of Trop and Med Hyg., 2002; 60: 109-118.

[18] Moody, A. H and Chiodini, P. L Methods for the detection of blood parasites Clin Lab of Heam, 2000; 22: 189-201.

[19] Tjitra, E., Suprianto, S., McBroom, J., Curie, B. J and Anstey, N. M Persistent ICT malaria P.f/P.v panmalarial and HRP2 antigen reactivity after treatment of Plasmodium falciparum malaria is associated with gametocytemia and results in false positive diagnosis of Plasmodium vivax in convalescence $J$ of Clin Microb., 2001; 39: 1025-1031.

[20] Murray, C. K and Benneth, J. W Rapid Diagnosis of Malaria Interdisc Perspec of Infect Dis., 2009; 320: 120-133.

[21] Moody, A. H and Chiodini, P Non microscopic method for malaria diagnosis using OptMAL IT, a second-generation dipstick for malaria pLDH antigen detection $\mathrm{Br} J$ of Biomed Sci., 2002; 59: 228-231.

[22] Berzosa, P., De Lucio, A., Romay-Barja, M., Herrador, Z., González, V., García, L., Fernández-Martínez, A., SantanaMorales, M., Ncogo, P., Valladares, B., Riloha M and Benito, A Comparison of three diagnostic methods (microscopy, RDT, and PCR) for the detection of malaria parasites in representative samples from Equatorial Guinea Mal J., 2018; 17: 333 .

[23] Askling, H H., Bruneel, F., Burchard, G., Castelli, F., Chiodini, P. L., Grobusch, M. P., Lopez-Vélez, R., Paul, M., Petersen, E., Popescu, C., Ramharter, M and Schlagenhauf, P Management of imported malaria in Europe Mal J., 2012; 11: 328.

[24] Mfuh, K. O., Achonduh-Atijegbe, O. A., Bekindaka, O. N., Esemu, L. F., Mbakop, C. D., Gandhi, K., Leke, R. G. F., Taylor, D. W and Nerurka, V. R A comparison of thick-film microscopy, rapid diagnostic test, and polymerase chain reaction for accurate diagnosis of Plasmodium falciparum malaria Mal J., 2019; 18: 73. 